\title{
Ubiquitin-Specific-Processing Protease 47, A Novel 53BP1 regulator
}

\author{
Doraid T. Sadideen ${ }^{1,2}$, Baowei Chen ${ }^{2}$, Manal Basili², Montaser Shaheen ${ }^{2,3,4}$
}

${ }^{1}$ Cancer Biology Graduate Interdisciplinary Program, The University of Arizona, Tucson, AZ 85724, USA.

${ }^{2}$ University of Arizona Cancer Center, The University of Arizona, Tucson, AZ 85724, USA

${ }^{3}$ College of Medicine, The University of Arizona, Tucson, AZ 85724, USA

${ }^{4}$ Corresponding Author: Montaser Shaheen, University of Arizona, 1515 N. Campbell

Avenue, Tucson, AZ 85724. Phone: (520) 626-0501; E-mail: shaheenm@arizona.edu

\begin{abstract}
DNA double strand breaks (DSBs) are repair by homology-based repair or non-homologous end joining and multiple sub-pathways exist. 53BP1 is a key DNA double strand break repair protein that regulates repair pathway choice. It is key for joining DSBs during immunoglobulin heavy chain class switch recombination. Here we identify USP47 as a deubiquitylase that associates with and regulates 53BP1 function. USP47 loss results in 53BP1 instability in proteasome dependent manner, and defective 53BP1 ionizing radiation induced foci (IRIF). USP47 catalytic activity is required for maintaining 53BP1 protein level. Similar to 53BP1, USP47 depletion results in sensitivity to DNA DSB inducing agents and defective immunoglobulin CSR. Our findings establish a function for USP47 in DNA DSB repair at least partially through 53BP1.
\end{abstract}

\section{Introduction}

DNA damage occurs due to a number of internal and external insults andsophisticated repair machineries have evolved to repair the damaged DNA and to maintain Genome 
instability.DNA damage can manifest in different forms and DNA double strand breaks (DSBs) are some of the most toxic to eukaryotic cells and can lead to cellular death or if non-or misrepaired to genomic instability and cancer ${ }^{1}$. There are a number of DSB repair pathways that are divided in general into two major categories: Homologous recombination (HR) and nonhomologous end joining. $\mathrm{HR}$ is operational during $\mathrm{S}$ and $\mathrm{G} 2$ phases of cell cycle when replicated DNA exists for homology search². Multiple proteins arerecruited to the damage site to activate the DNA damage response and to regulate the DSB end processing and channel the repair to one of these repair pathways ${ }^{3}$.

TP53 Binding protein 1 (53BP1) is a key scaffold protein that is recruited early to the repair process and help determine the pathway choice ${ }^{4}$. Thousands of 53BP1 molecules build at the damage site through specific chromatin interaction and oligomerization to form a visible Ionization irradiation induced foci (IRIF). 53BP1 recruits a machinery that antagonizes DNA DSB end resection and blocks HR through a pre- and post- resection mechanisms ${ }^{5}$. These 53BP1 foci last for a few hours and ultimately dissolve by an unknown mechanism. A large number of proteins associate with 53BP1 and likely contribute to the repair process through yet to be uncovered mechanisms ${ }^{6}$. Ubiquitin related processes are abundant at the repair sites although the functions of several ubiquitin-associated proteins keep evolving or remain unknown ${ }^{7}$.

USP47 is one of about 100 human deubiquitylases (dubs) that regulate thousands of ubiquitylated events that involve most cellular proteomes in human ${ }^{8}$. It was reported to be involved in Base Excision Repair (BER) through interacting with and deubiquitylating thepolymerase Pol $\beta^{9}$. USP47 has significant homology to USP7, a deubiquitylase that is involved in a plethora of chromatin and DNA damage processes ${ }^{10}$.

We searched for novel 53BP1 Binding proteins that may regulate its function in DSB repair and identified USP47 as a novel 53BP1 regulator. 


\section{Results}

\section{USP47 associate with 53BP1 after DNA damage}

To search for novel 53BP1 binding proteins post DNA damage, we performed immuneprecipitation utilizing a high affinity antibody that binds endogenous 53BP1. We subjected the immune-precipitates to mass spectrometry fingerprinting and we identified a number of proteins that existed in 53BP1 Pull down but not in multiple other control pull-downs from our lab and from other non-specific binders reported in literature. We identified known 53BP1 partners such as RIF1, and laminB1. In addition, USP47 was recovered in the 53BP1 IPs and not from control (Figure 1A).

We did 53BP1 IP followed by WB and confirmed the two proteins association utilizing endogenous protein pull-down (Figure 1B) and another IP on Turbo-GFP trap after tGFP-53BP1 expression (Figure 1C). As noted in Figure 1B\& $\mathrm{C}$, the two proteins association is enhanced after exposing cells to IR. While USP47 did not form IRIF upon IR exposure, however, we show that the two protein colocalize on a cluster of DNA DSBs trigged by FOk1 nuclease, taking advantage of the more sensitive Lacl-Fok1 system (Figure 1D).

\section{USP47 regulates 53BP1 Stability through deubiquitylation}

A large-scale screen of ubiquitin pathway proteins' impact on 53BP1 focus generation or persistence identified key proteins that are required for 53BP1 IRIF, such as the ubiquitin E3 ligase $\mathrm{RNF} 168^{11}$. The proteasome proteins scored highest positive Z-score in this screen suggesting that the proteasome plays a key role in antagonizing 53BP1 focus formation. We hypothesized that USP47 depletion can lead into 53BP1 protein instability, and indeed, reducing USP47 protein level by siRNA or through CRISPR knock-out is associated with a decrease in 53BP1 protein levels particularly after IR (Figure 2A, B, C). 53BP1 protein level declines faster in USP47 -/- cells after halting new protein synthesis consistent with reduced half-life after IR 
(Figure 2D). This decline in 53BP1 was at least partially reversed after proteasome inhibition consistent with a proteasome mediated degradation (Figure 3A, B). We looked at 53BP1 ubiquitylation status in human cell lines after proteasome inhibition, and with lysate collection under denaturing conditions to preserve ubiquitylated species. We observed enhanced 53BP1 high molecular weight ubiquitylated species in USP47 deficient cells consistent with enhanced ubiquitylation (Figure 3C). WT USP47 but not catalytically dead mutant was able to reverse 53BP1 protein instability (Figure 3D)

\section{USP47 depletion impairs 53BP1 recruitment and function}

Having established an impact of USP47 on 53BP1 protein levels, we investigated in this depletion affects its function in DNA DSB repair. First, we observed significant decline in 53BP1 recruitment to damage sites after USP47 depletion (Figure 4). 53BP1 deficient cells are sensitive to DNA damaging agents that generate DSBs ${ }^{4}$. Similarly, we observed radiation and cisplatin sensitivity to cells depleted of USP47 compared to control isogenic cells (Figure 5A, B). A major function of 53BP1 is to facilitate long range DSB repair of cuts generated during immunoglobulin heavy chain class switch recombination $(\mathrm{CSR})^{12}$. Some believe 53BP1 evolved to facilitate this process ${ }^{4}$, and 53BP1 deficiency leads to a dramatic CSR defect. We investigated USP47 contribution to CSR through a murine lymphocytic cell line that class switch upon cytokine stimulation. We depleted USP47 by lentivirus shRNA, and we identified significant impairment in CSR although not to the same profound impairment that is seen with 53BP1 total loss by CRISPR (Figure 5C, D).

\section{Discussion}

We identify here another protein that contributes to DNA DSB through at least interacting with and stabilizing 53BP1. Consistent with a DNA repair function USP47 is phosphorylated at defined residues post IR and etoposide ${ }^{13}$. USP47 was previously implicated in another DNA 
repair pathway $(B E R)$ by stabilizing the polymerasePol $\beta^{9}$. Since some DNA repair proteins function in multiple pathways, it is not surprising that USP47 operates in two distinct pathways. As a deubiquitylase, it probablyhandles dozens of substrates given limited number of deubiquitylases that have to regulate all the ubiquitylated proteome. FEN1 is another proteinthat function in both BER and DSB through microhomology mediatedend-joining (MMEJ $)^{14}$. It is interesting that large scale proteomics reveal an association between FEN1 and USP47, and FEN1 and 53BP1 $1^{15,16}$. One of these proteomic studies also show an association between USP47 and MDC1 $1^{15,16}$, a major partner of 53BP1.

53BP1 regulation byubiquitin and ubiquitin like proteins is likely extensive given the number ofubiquitin and sumo associated proteins that are discovered in proximity to $53 \mathrm{BP} 1^{6}$. Future research will likely identify which ubiquitin ligase destine 53BP1 to the proteasome and will further shed light into other hidden substrates/ functions of USP47. 


\section{Figures}

A)

\begin{tabular}{|c|}
\hline 53BP1 \\
\hline Lamin B1 \\
\hline PGAM5 \\
\hline ALYREF \\
RIF1 \\
C18ORF63 \\
USP47
\end{tabular}

D)

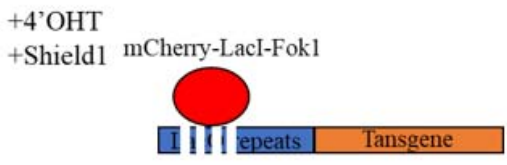

53BP1
B) U2OS

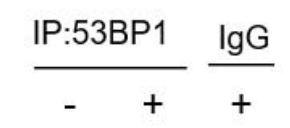

USP47

53BP1

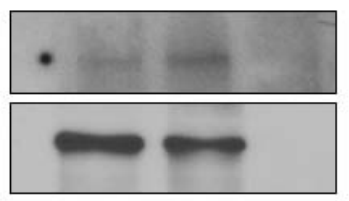

Input

USP47

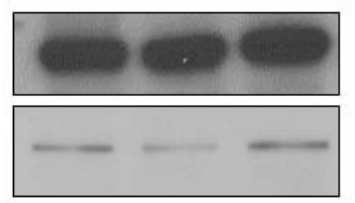

C) U2OS

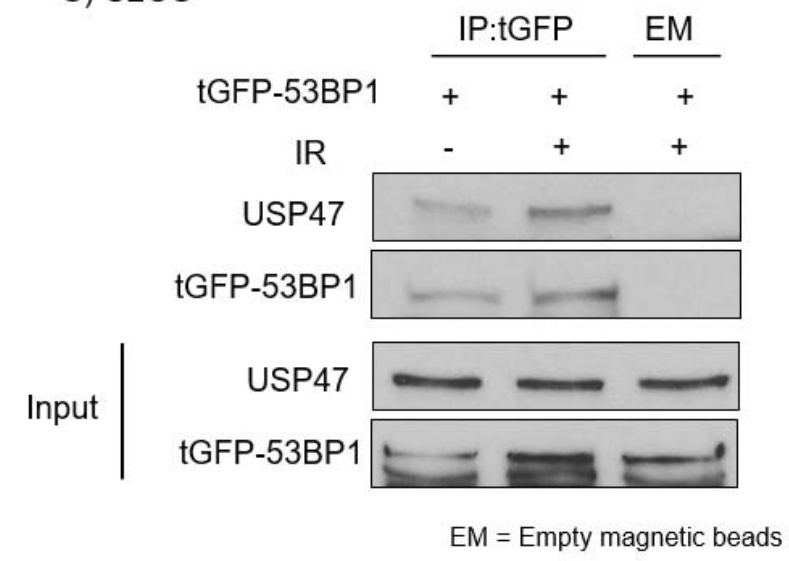

E)

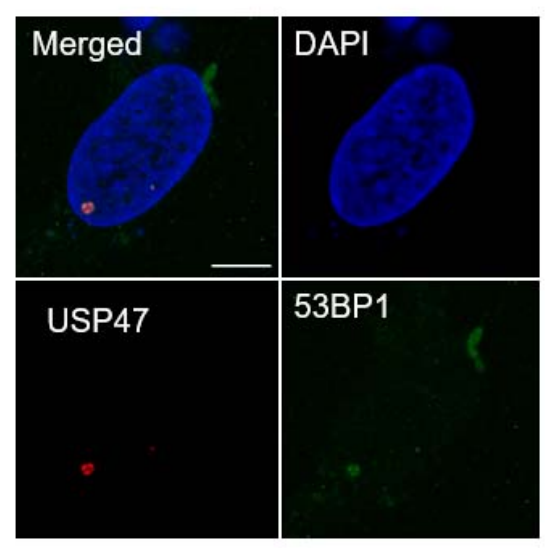

Figure 1: USP47 is a novel 53BP1 interacting partner.

A.Selected lists of proteins associated with 53BP1 analyzed by mass spectrometry after pulldown using 53BP1 high affinity antibody. B.Immunoprecipitation of USP47 using high affinity antibody against endogenous 53BP1. C. Pull-down of USP47 after overexpression of tGFP53BP1 in U2OS cells. D.Schematic representation of Fok1 DSB-reporter cell line. E.Fok1 DSBreporter cell line transfected with tGFP-53BP1 and treated with 4-OHT and Shield1 for 4 hrs to induce DSBs by mCherry-Lac1-FokI. USP47 co-localizes with 53BP1 on DSBs locus. Scale bar is $20 \mu \mathrm{m}$. 


\section{A) U2OS}

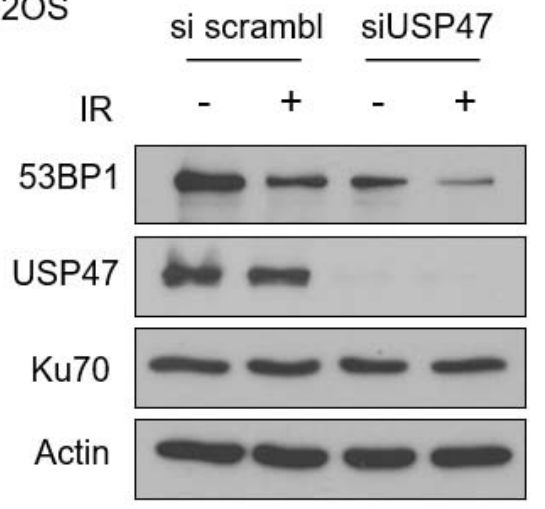

B) A549

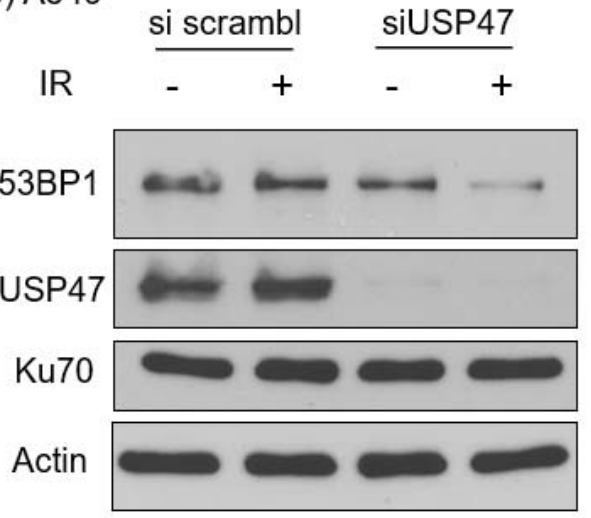

C) A549

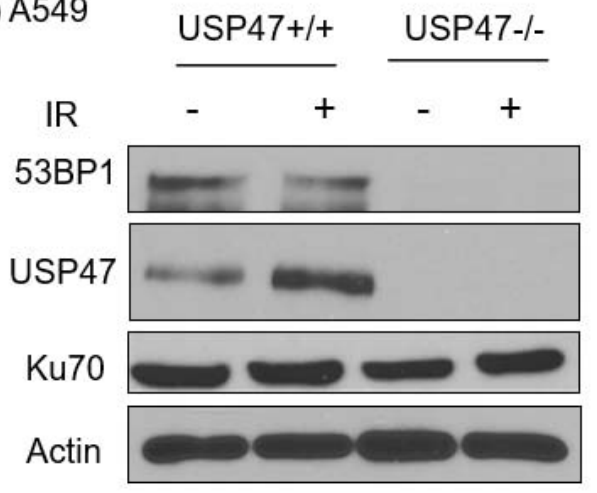

D) A549
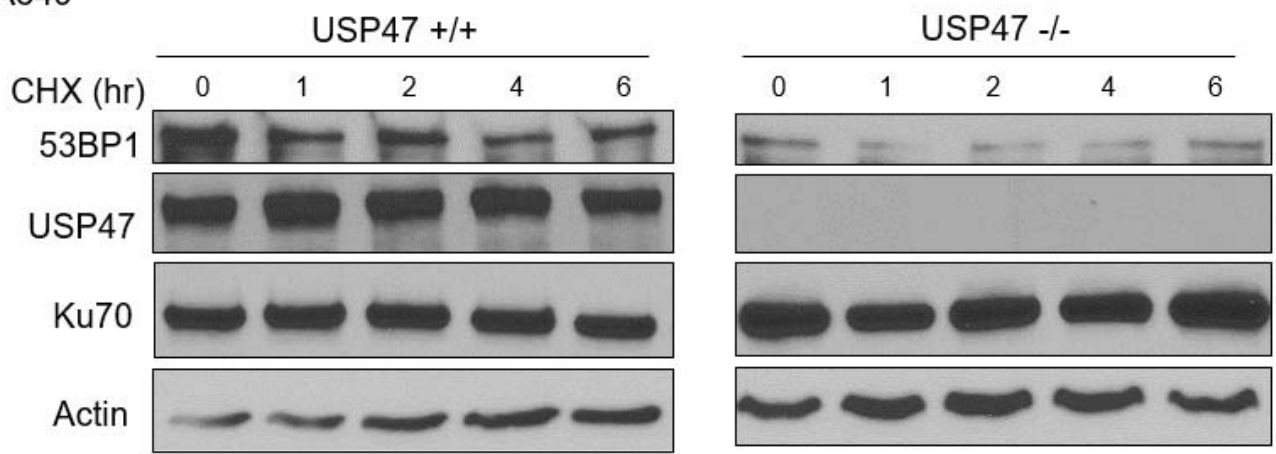

Figure 2: USP47 regulates 53BP1 Stability through deubiquitylation.

A-B. Transient knockdown of USP47 through siRNA in U2OS and A549 cells, respectively. C. USP47 CRISPR-generated A549 knockout cell line. D. A549 proficient and deficient USP47 cells treated with Cycloheximide and collected after IR after indicated time points. 
A) U2OS

$\begin{array}{ccccc}\text { Si Scramble } & + & - & + & - \\ \text { Si USP47 } & - & + & - & + \\ \text { IR } & + & + & + & + \\ \text { Carfilzomib } & - & - & + & +\end{array}$

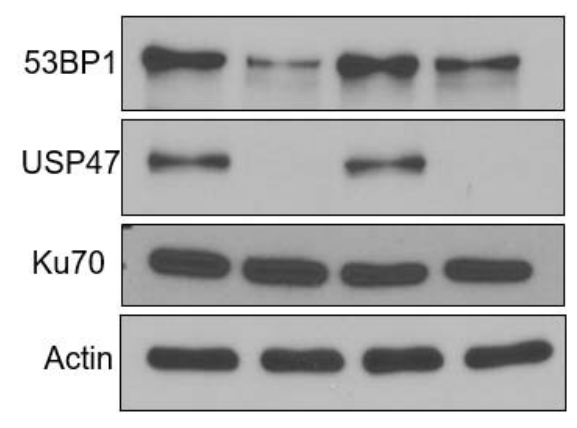

C) A549

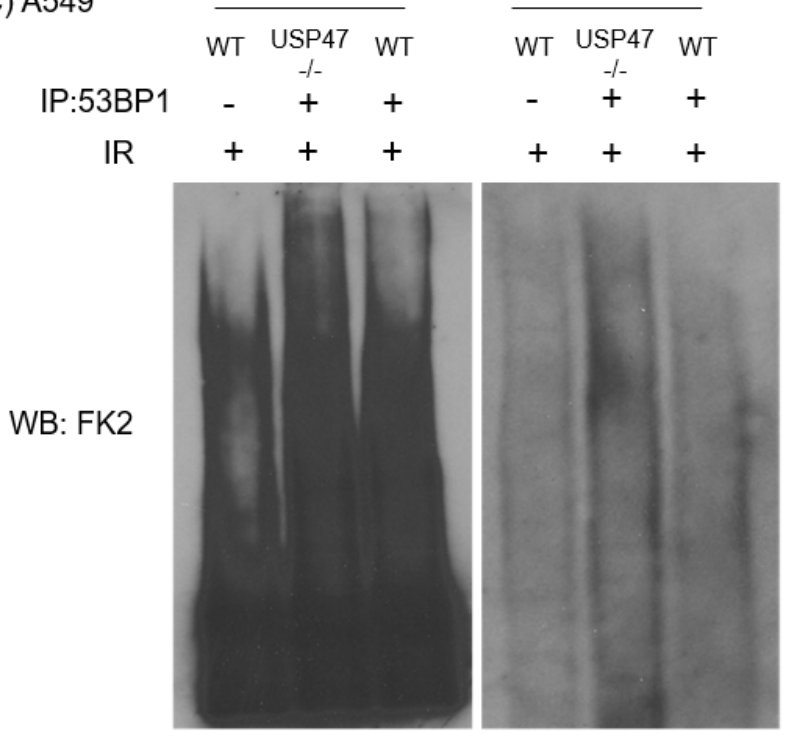

B) $A 549$

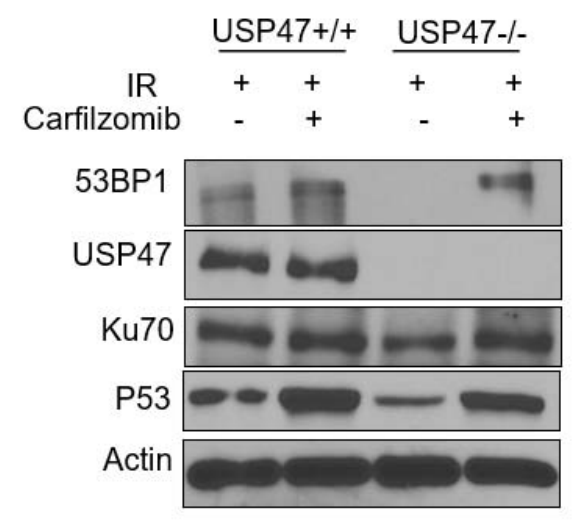

D) A549

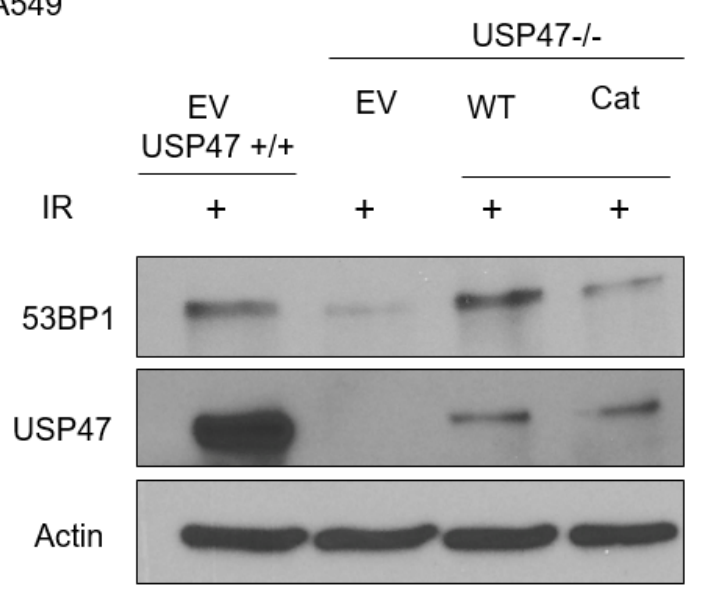

Figure 3: USP47 antagonizes proteasome degradation of 53BP1.

A. siRNA Transient knockdown of USP47 U2OS cells leads to increase instability of 53BP1. B. CRISPR-generated USP47 A549 knockout cell line shows a dramatic phenotype to A. C. Enhanced ubiquitylation of 53BP1 in USP47 -/- cells compared to USP47+/+ cells. D. Overexpression of wild-type USP47 rescues 53BP1 instability, while catalytically dead USP47 does not in CRISPR-generated USP47 deficient cells. 
A)

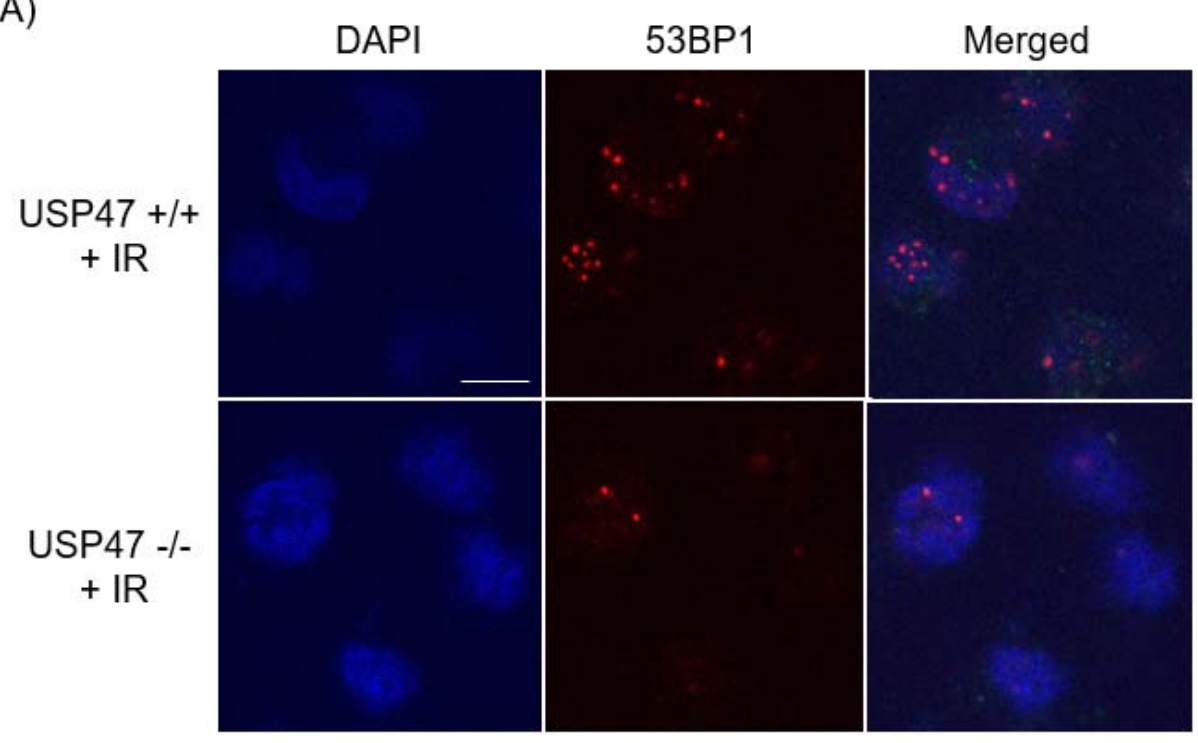

B)

USP47 Impact 53BP1 Foci Formation

C)

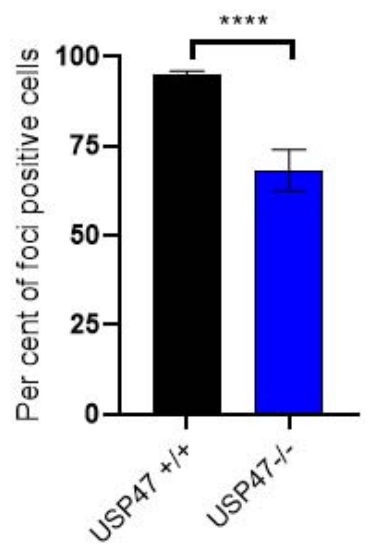

USP47 +/+

USP47-I-
USP47 Impact 53BP1 Foci Intensity

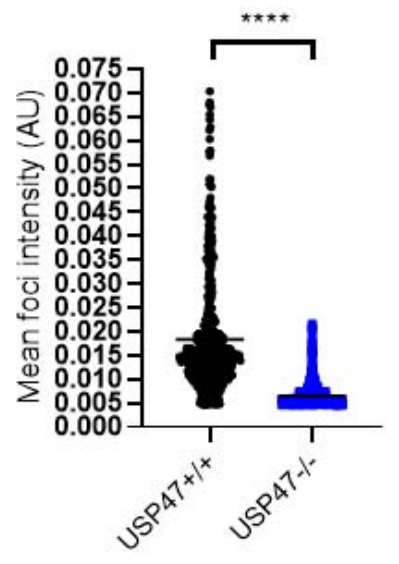

- USP $47+1+$

- USP47-/-

Figure 4: 53BP1 decline recruitment to DNA DSB foci in USP47 deficient cells.

A. A549 CRISPR-generated USP47 proficient and deficient cells show drastic difference between 53BP1 foci formation after IR exposure. B. Quantification of 53BP1 foci in USP47 +/+ and USP47 -/- cells after 4hrs of IR exposure. C. Measurement of 53BP1 foci intensity in USP47 +/+ and USP47 -/- cells after $4 \mathrm{hrs}$ of IR exposure. Scale bar is $10 \mu \mathrm{m}$. 
A)

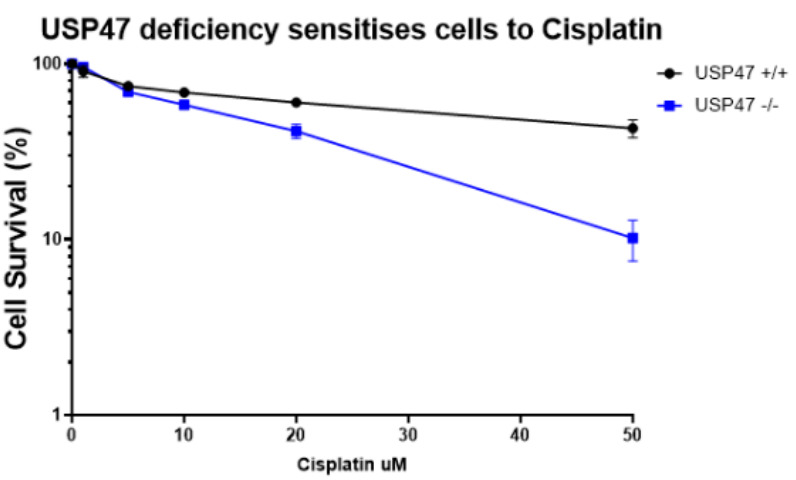

B)

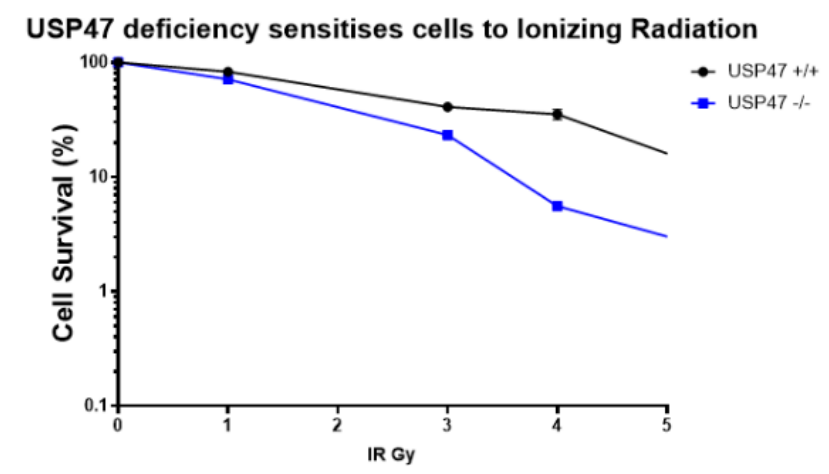

C)

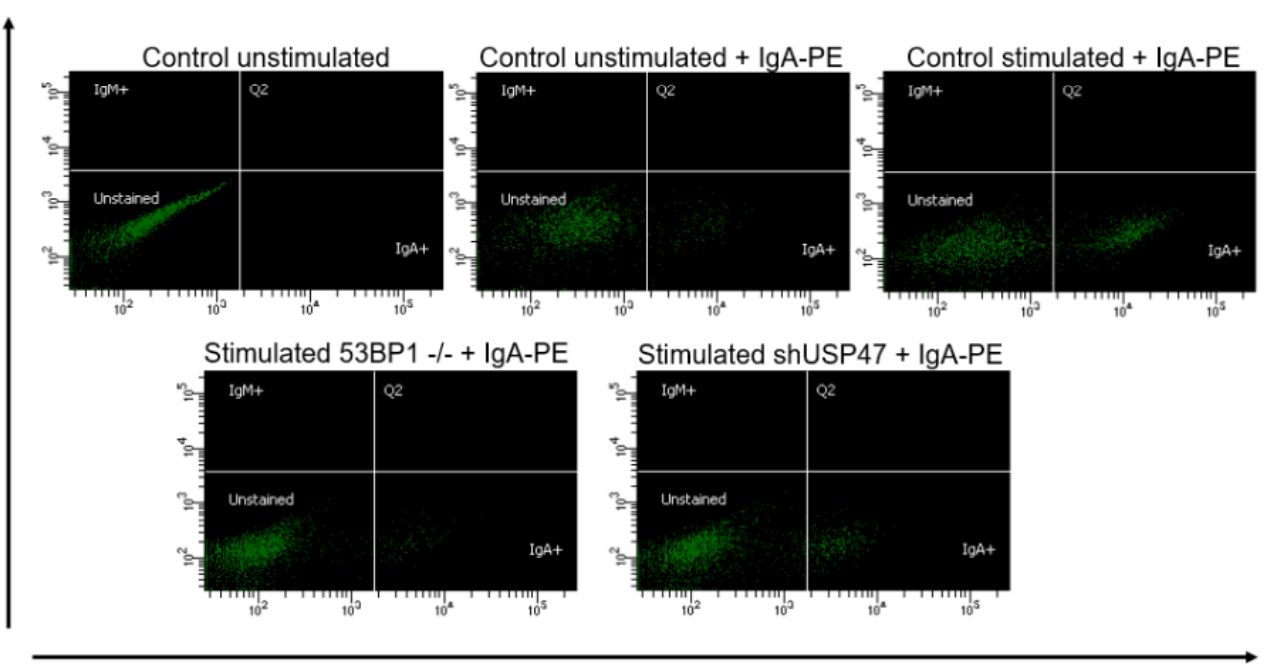

D)

USP47 deficiency impairs CSR in $\mathrm{CH} 12 \mathrm{~F} 3$ cells

PE-A

Figure 5: USP47 deficiency affects 53BP1 function.

A. USP47 proficient and deficient cells exposed to indicated IR dose and left to grow for 14 days. B. Similar to $\mathbf{A}$ except cells treated with Cisplatin at indicated dosages. C. Flow cytometry plots measuring CSR to IgG with indicated shRNA targeted gene suppression. D. Quantification of flow cytometry plots from $\mathbf{C}$. 


\section{Materials and Methods}

\section{Cell Lines, CRISPR and Gene Silencing}

Human HEK293 (ATCC, CRL-1573), A549 (ATCC, CRM-CCL-185) and U2OS (ATCC, HTB-96) cells were maintained in Dulbecco's Modified Eagle Medium (DMEM, Corning) supplemented with $5 \%$ heat-inactivated fetal bovine serum (FBS) (Genesee Scientific) in a $5 \%$ $\mathrm{CO} 2$ incubator at $37^{\circ} \mathrm{C}$. CH12F3-2 and CH12F3-2 53BP1-/- murine B cell lymphoma cells ${ }^{17,18}$ were cultured in RPMI 1640 medium supplemented with 15\% FBS, 50nM 2-Mercaptoethanol (BME), 5\% NCTC-109 (Life Technologies)and 20 mM HEPES (Corning).

Short-term depletion of USP47 expression was achieved by treating cells with siRNA USP47 (5'-GAA AGC AAA UGA AGG GAA AUU-3' or 5'-AGA UAA AGA UGG UGA ACA AUU3') from Dharmacon and Lipofectamine RNAiMAX transfecting reagent twice according to the manufacturer's instruction (Invitrogen). All siRNAs were purchased from Dharmacon unless otherwise stated. Other siRNA used in the study are:

\section{BP1: Sense: 5'-GAA CAG AAG UAG AAA GAA AUU-3'}

Scramble from Qiagen Cat No.: 1027310: Sense: 5'-UUC UCC GAA CGU GUC ACG UdT dT-3'

To generate CRISPR deficient USP47 cell line transfection was performed according to IDT's instruction manual with modification. Briefly, using reverse transfection, A549 cells were trypsinized and plated $5 \times 10^{\wedge} 5$ cells per well in 6 -well plate mixed with $800 \mu \mathrm{L}$ of RNP complex reverse transfection and crRNA sequence are 5'-ACTGTCATCACGGAGTACAA-3' and 5'GCAATATAGTAGAAACGAGT-3' or proprietary non-targeting sequence for control. After 24hrs, the complex was removed and fresh DMEM supplemented with $10 \%$ FBS was added. The cells were trypsinized 48hrs later and sorted through FACS in 96-well plates for colonies. Two week later, the 96-well plates were screened for homozygous knockout of USP47.

\section{Reagents and Plasmids}

The proteasome inhibitors MG132 and Carfilzomib were purchased from Sigma-Aldrich and TLC Pharmaceutical Standards Ltd., respectively. Protein synthesis inhibitor cycloheximide was purchased from Sigma-Aldrich. USP47 catalytic domain was mutated by changing cysteine 177 into alanine (C177A) using the sense primer (5'-

gtttgcaaaaggctattcaaataggcagtcattgcttggtttactagtcc-3') and Quickchange Lightning Site-directed Mutagenesis Kit from Agilent technologies.

\section{Western Blotting, Immunoprecipitation and Antibodies}

Cell were washed with PBS (137mM NaCl, $2.7 \mathrm{mM} \mathrm{KCl,} \mathrm{10mM} \mathrm{Na} 2 \mathrm{HPO}_{4}, 1.8 \mathrm{mM}$ $\mathrm{KH}_{2} \mathrm{PO}_{4}$ ) then collected $4 \mathrm{hrs}$ after irradiation at a single dose of $6 \mathrm{~Gy}$ using a Cobalt-60 Source Gamma Irradiator $72 \mathrm{hrs}$ after siRNA exposure in lysis buffer $(150 \mathrm{mM} \mathrm{NaCl}, 50 \mathrm{mM}$ Tris- $\mathrm{HCl} \mathrm{pH}$ 8, 1mM Dithiothreitol (DTT), 0.5\% NP-40, with protease and phosphatase inhibitors (Sigma)), sonicated for 10 seconds twice, and centrifuged at $4^{\circ} \mathrm{C}$ at $14 \mathrm{Kxg}$ for $1 \mathrm{~h}$. Protein quantification performed using Bradford Protein Assay (Bio-Rad), and $20 \mu \mathrm{g}$ samples were resolved on a $7 \%$ SDS-PAGE gels, transferred to nitrocellulose or PVDF membranes, blocked with $5 \%$ non-fat milk or $2 \%$ BSA then incubated with primary antibodies overnight. Horseradish peroxidase 
secondary antibodies and enhanced chemiluminescence (ECL) substrate from Santa Cruz Biotechnology were used to detect antigen-antigen interaction.

Cells were collected with IP lysis buffer (150mM NaCl, 50mM Tris- $\mathrm{HCl} \mathrm{pH} 8,1 \mathrm{mM}$ EDTA, $1 \mathrm{mM}$ DTT, $0.5 \% \mathrm{NP}-40$, with protease and phosphatase inhibitors (Sigma)), sonicated for 10 seconds twice at $60 \mathrm{~Hz}$, and centrifuged at $4^{\circ} \mathrm{C} 14 \mathrm{Kx} \mathrm{g}$ for $1 \mathrm{hr}$. Samples were pre-cleared using protein $\mathrm{G}$ magnetic beads (Dynabeads, Invitrogen). The lysate then incubated overnight with beads conjugated with corresponding antibodies. The beads were washed four times with IP lysis buffer and four times with PBS then boiled in Laemmli sample buffer.

Antibodies include rabbit anti-53BP1 (ab36823, Abcam and NB100-304, Novus Biologicals), rabbit anti-USP47 (A301-048A, Bethyl Laboratories), mouse anti- $\beta$-actin (A2228, Sigma), mouse anti-FK2 (04-263, Sigma), mouse anti-TurboGFP (TA150041, Origene), mouse anti-FLAG M2 (F1804, Sigma) and mouse anti-GFP (sc-9996, Santa Cruz).

\section{Immunofluorescence}

Cells grown on coverslips were exposed to $1 \mathrm{~Gy}$ IR. After $4 \mathrm{hrs}$, the cells were fixed for 10 minutes in $4 \%$ formaldehyde and sucrose at room temperature. Then permeabilized for 10 minutes in $0.5 \%$ Triton-X in PBS and blocked for $1 \mathrm{hr}$ in $4 \%$ BSA in PBS at room temperature. The cells were washed three times with PBS before every step. The indicated primaries were diluted in 1\% BSA in PBS, while secondary antibodies were diluted in PBS. Both incubated at room temperature for $1 \mathrm{hr}$.

\section{Clonogenic Assay}

CRISPR-generated USP47 cells were plated at approximately 500 cells per well in 6well plate or $60 \mathrm{~mm}$ dishes. The following day, cells were incubated with cisplatin for $2 \mathrm{hrs}$ at indicated concentrations then washed three times with PBS and fresh medium was add to the wells or exposed to IR at indicated dosages and left to grow. After 14 days, colonies (defined as $>50$ cells) were fixed and stained with $1 \%$ Crystal violet solution (Sigma).

\section{In Vivo Deubiquitylation}

For in vivo deubiquitylation of 53BP1 by USP47, A549 CRISPR-generated USP47 proficient and deficient cells were used to overexpress tGFP-53BP1 using Lipofectamine 2000 according to manufacturer's instructions. 48hrs after transfection, cells were exposed to 6Gy IR dose and treated with $500 \mathrm{uM}$ Carfilzomib for $4 \mathrm{hrs}$. Cells were collected in lysis buffer (150mM $\mathrm{NaCl}, 50 \mathrm{mM}$ Tris-HCl pH 8, $1 \mathrm{mM}$ DTT, $0.5 \% \mathrm{NP}-40$, with protease and phosphatase inhibitors (Sigma)).After $1 \mathrm{~h}$, the reaction was stopped by boiling in SDS-based loading buffer, and the ubiquitylated species was detected by anti-ubiquitin chain FK2 antibody.

\section{Class switch recombination assay}

CH12F3-2 and CH12F3-2 53BP1-/- murine B cell lymphoma cells were treated with either shScramble or shUSP47. For induction of CSR, CH12F3-2 cells were incubated in culture medium supplemented with10ng/mL IL-4 (R\&D Systems \#404-ML-050), 1ng/mL TGFß (R\&D Systems \#7666-MB-005) and 1 $\mathrm{gg} / \mathrm{mLanti-CD40}$ antibody (Thermo Fisher\#16-0401-86) for 48hrs. Anti-lgA-PE was used to stain the cells and subsequently PE signal analyzed on BD FACSCanto II machine.

\section{Statistical analysis}


All results are from, at least, three independent experiments. Student's t-test was used to compare two groups, while one-way analysisofvariance(ANOVA) for comparing more than two groups. Graphs were generated using GraphPad Prism 8.0.2 on Microsoft Windows 10 (La Jolla, CA, USA). P-value less than 0.05 will be considered statistically significant. 


\section{References}

1. Kass EM, Moynahan ME, Jasin M. When Genome Maintenance Goes Badly Awry. Mol Cell. 2016 Jun 2;62(5):777-87.

2. Hustedt N, Durocher D.The control of DNA repair by the cell cycle .Nat Cell Biol. 2016 Dec 23;19(1):1-9.

3. Wilson MD, Durocher D. Reading chromatin signatures after DNA double-strand break Philos Trans R Soc Lond B Biol Sci. 2017 Oct 5;372(1731):20160280

4. Mirman Z, de Lange T. 53BP1: a DSB escort. Genes Dev. 2020 Jan 1;34(1-2):7-23

5. Callen E, Zong D, Wu W, et al. 53BP1 Enforces Distinct Pre- and Post-resection Blocks on Homologous Recombination. Mol Cell. 2020 Jan 2;77(1):26-38.e7.

6. Gupta R, Somyajit K, Narita T, et al. DNA Repair Network Analysis Reveals Shieldin as a Key Regulator of NHEJ and PARP Inhibitor Sensitivity. Cell. 2018 May 3;173(4):972-988.e23

7. Jackson SP, Durocher D. Regulation of DNA damage responses by ubiquitin and SUMO. Mol Cell. 2013 Mar 7;49(5):795-807

8. Akimov V, Barrio-Hernandez I, Hansen SVF, et al. UbiSite approach for comprehensive mapping of lysine and N-terminal ubiquitination sites Nat Struct Mol Biol. 2018 Jul;25(7):631640

9. Parsons JL, Dianova II, Khoronenkova SV, et al. USP47 is a deubiquitylating enzyme that regulates base excision repair by controlling steady-state levels of DNA polymerase $\beta$. Mol Cell. 2011 Mar 4;41(5):609-15.

10. Valles GJ, Bezsonova I, Woodgate R, et al. USP7 Is a Master Regulator of Genome Stability. Front Cell Dev Biol. 2020 Aug 5;8:717 
11. Moudry P, Lukas C, Macurek L, et al. Nucleoporin NUP153 guards genome integrity by promoting nuclear import of 53BP1. Cell Death Differ. 2012 May;19(5):798-807.

12. Manis JP, Morales JC, Xia Z, et al. 53BP1 links DNA damage-response pathways to immunoglobulin heavy chain class-switch recombination. Nat Immunol. 2004 May;5(5):481-7

13. Beli P, Lukashchuk N, Wagner SA, et al: Proteomic investigations reveal a role for RNA processing factor THRAP3 in the DNA damage response. Mol Cell. 2012 Apr 27;46(2):212-25.

14. Mengwasser KE, Adeyemi RO, Leng $\mathrm{Y}$, et al. Genetic Screens Reveal FEN1 and APEX2 as BRCA2 Synthetic Lethal Targets. Mol Cell. 2019 Mar 7;73(5):885-899.e6

15. Huttlin EL, Bruckner RJ, Paulo JA, et al. Architecture of the human interactome defines protein communities and disease networks. Nature. 2017 May 25;545(7655):505-509.

16. chweppe DK, Huttlin EL, Harper JW, Gygi SP. BioPlex Display: An Interactive Suite for Large-Scale AP-MS Protein-Protein Interaction Data. J Proteome Res. 2018 Jan 5;17(1):722726

17. Nakamura M., Kondo K., Sugai M., Nazarea M., Imamura S., Honjo T. (1996) High frequency class switching of an IgM+B lymphoma clone $\mathrm{CH} 12 \mathrm{~F} 3$ to $\lg A+$ cellsInt. Immunol., 8 pp. 193-201.

18. Noordermeer S.M., Adam S., Setiaputra D., Barazas M., Pettitt S.J., Ling A.K., Olivieri M., Álvarez-Quilón A., Moatti N., Zimmermann M., Annunziato S., Krastev D.B., Song F., Brandsma I., Frankum J., Brough R., Sherker A., Landry S., Szilard R.K., Munro M.M., McEwan A., Goullet de Rugy T., Lin Z.Y., Hart T., Moffat J., Gingras A.C., Martin A., van Attikum H., Jonkers J., Lord C.J., Rottenberg S., Durocher D. The shieldin complex mediates 53BP1dependent DNA repair. Nature. 2018;560(7716):117-121. doi: 10.1038/s41586-018-0340-7. 
bioRxiv preprint doi: https://doi.org/10.1101/2021.01.27.428282; this version posted January 27, 2021. The copyright holder for this preprint (which was not certified by peer review) is the author/funder. All rights reserved. No reuse allowed without permission. 